This is a post-peer-review, pre-copyedit version of an article (Jakšić, O., Jokić, I., Jakšić, Z. et al. The time response of plasmonic sensors due to binary adsorption: analytical versus numerical modeling) published in Applied Physics A: Materials Science and Processing (Appl. Phys. A 126, 342, 2020) The final authenticated version is available online at: https://doi.org/10.1007/s00339-020-03524-3 
This is a postprint version of a paper published with SpringerNature in Applied Physics A Read and share here: https://rdcu.be/b3yxi

Jakšić, O., Jokić, I., Jakšić, Z. et al. The time response of plasmonic sensors due to binary adsorption: analytical versus numerical modeling. Appl. Phys. A 126, 342 (2020).

https://doi.org/10.1007/s00339-020-03524-3

\title{
The time response of plasmonic sensors due to binary adsorption: analytical vs. numerical modeling
}

\author{
Olga Jakšić ${ }^{1}$ Ivana Jokić ${ }^{1}$ Z Zoran Jakšić IVana Mladenović $^{1} \cdot$ Katarina \\ Radulović $^{1} \cdot$ Miloš Frantlović ${ }^{1}$
}

Received: date / Accepted: date

\begin{abstract}
In order to allow for multiscale modeling of complex systems we focus on various approaches to modeling binary adsorption. We consider multiple methods of modeling the temporal response of general plasmonic sensors. We start from the analytical approach. The kinetics of adsorption and desorption is modeled both as a first order reaction and as a second order reaction. The criteria for their validity and the choice between them in the case of two-component adsorption are established. Due to the nonlinearities of the second order reactions and the lack of their analytical solutions, computer aided modeling is considered next, also in multiple ways: the employment of numerical solvers, fitting of experimental results, the stochastic simulation algorithms and the employment of artificial neural networks (ANN). The examples we present illustrate the advantages and disadvantages of the particular approaches. The goal is to aid the concurrent multiscale modeling of adsorption-based devices. Machine learning in ANN performed here is used to estimate the equilibrium values of adsorbed quantities. The obtained results show that to train an ANN for the estimation of the equilibrium adsorption quantities the Levenberg-Marquardt and the Bayesian regularization algorithms are less efficient than the quasi-Newton BFGS (Broyden-Fletcher-GoldfarbShanno) algorithm.
\end{abstract}

Keywords adsorption $\cdot$ kinetics $\cdot$ machine learning algorithms $\cdot$ plasmonic sensing stochastic simulation algorithms

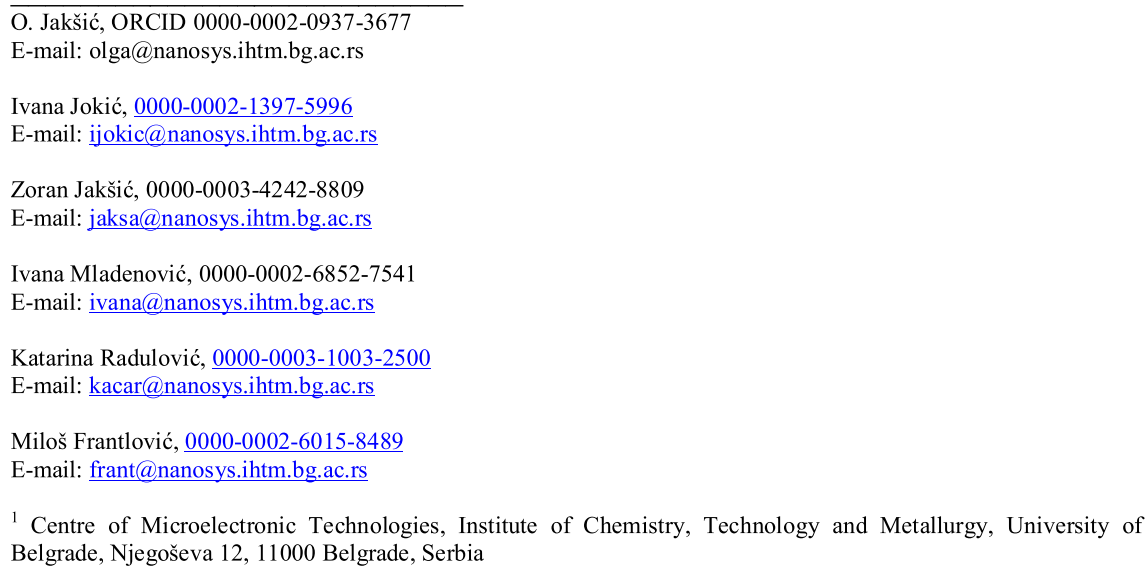

${ }^{1}$ Centre of Microelectronic Technologies, Institute of Chemistry, Technology and Metallurgy, University of Belgrade, Njegoševa 12, 11000 Belgrade, Serbia 


\section{Introduction}

Adsorption-desorption processes are employed in many industrial applications [1-4], but also in novel devices based on emerging technologies [5]. Adsorption-based devices with optical readout, such as plasmonic sensors, enable the detection of the targeted species but also the investigation of the kinetics of the adsorption process itself.

Early empirical approaches to the kinetics of adsorption processes had resulted in a first order differential equation modeling the exponential transition of the number of the adsorbed molecules from an initial value to a steady-state value [6,7], like in the first order reactions. Starting from the stoichiometric equation valid for the binary adsorptiondesorption one gets analytical models in a form of coupled nonlinear second order Riccati differential equations $[8,9]$. They can be reduced to first order differential equations if certain conditions are met $[10,11]$. Novel models emerge with the advances of computing concepts and algorithms. They include those based on trained artificial neural networks (ANN) $[12,13]$. The prominent use of ANN in modeling allows for obtaining fast and reasonably reliable results that can be used independently or incorporated in a combined multiscale approach to modeling [14]. Trained on ab initio calculations or experimental results, ANNs can be used for providing constants for the kinetic parameters, such as desorption energy - otherwise determined experimentally or by density functional theory calculations [15] - or for providing kinetic parameters themselves.

It is documented that ANNs can be used for the estimation of reaction rate constants in chemical kinetics [16,17], for predicting adsorbed amounts of copper using ground nut seed cake powder, sesame seed cake powder and coconut cake powders as bio adsorbents [18] or for the estimation of time degradation of adsorbate in binary mixtures exhibiting linear Langmuir kinetics [19]. The employment of ANN is usually a part of multiscale analysis, like in [20], where a combination of molecular simulation and machine learning was used to examine the role of various pore topological structures on the $\mathrm{CO}_{2}$ capture capabilities of MOFs (metal organic frameworks). In [21] intelligent optimization algorithms were implemented for finding the optimum values for the adsorption of methylene blue dye by carob powder used for cleaning wastewater (particle size, temperature, solution concentration, contact time...). The use of machine learning classification algorithms for the optimization of methylene blue adsorption on silver nanoparticles attached to activated carbon is addressed in [22] while the computational modeling of the related adsorption process is investigated in references therein. An ANN, feed forward back-propagation neural network (FFBPNN), and Levenberg-Marquardt (L-M) training algorithm were applied for predicting the adsorption capacity and removal/uptake percentage of $\mathrm{Pb}$ (II) ions by rice husks as bio-adsorbents in [23]. A recent work on modelling of adsorption of methane, nitrogen, carbon dioxide, their binary mixtures, and their ternary mixture on activated carbons using an artificial neural network in [24] gives a good review of published works on the use of machine learning algorithms for modeling adsorption capacity. It also presents an example of using artificial neural network, trained on a set of 1440 experimental data sets, for modelling of adsorption of gases on activated carbons. Although targeting a broad application range, namely adsorption of methane, nitrogen, carbon dioxide, their binary mixtures, and their ternary mixture, the results in [24], as the work in before mentioned publications, still are application-specific. The published research clearly shows that the use of machine learning for the prediction of adsorbed amounts in various applications is feasible and that the results reach a high accuracy. However, it also shows that the analytical models are still at an advantage when it comes to the prediction in adsorption-based devices for an arbitrary analyte or mixture of analytes with known parameters. 
Analytical models of the temporal response of adsorption-based plasmonic sensors, valid for linear systems with the first order kinetics, are presented in [25]. Analytical models of the temporal response of adsorption based plasmonic sensors valid for nonlinear systems with second order kinetics of adsorption process are also known [26], both for monocomponent and multicomponent analyte environments [27]. The analytical results related to the linear systems are general and can be applied for the deterministic or stochastic analysis of performances of plasmonic sensors surrounded by a mixture of an arbitrary number of analytes [25]. The analytical approach, i.e. the theoretical analysis performed in [25] resulted in a probability-generating function providing one with an insight into all of the higher moments and statistical parameters of the process.

The results related to nonlinear systems are known in analytical form for monocomponent adsorption only. The calculations of the response of plasmonic sensors caused by multicomponent adsorption must then rely on the use of numerical ordinary differential equations (ODE) solvers. They may be stiff if some species have much smaller time scales than others, and consequentially, their solution may be time consuming or doubtful in that case. Knowing the way to simplify the problem by linearization for instance would be beneficial for faster comprehension of the dynamic behavior of the system. Knowing the criteria for the domain of applicability of the results based on the use of linear model would be even more beneficial since the stochastic behavior of such linear systems can be deduced from the deterministic response, as described in [25].

The criteria for validity of the pseudo-first-order kinetics in biochemical kinetics of ligand-receptor binding are investigated in [10]. The criteria for the domain of applicability of equations governing the response of a plasmonic sensor based on the adsorption process with the kinetics of the first order are known in analytical form $[11,26]$. The mentioned criteria refer to applications where the monocomponent adsorption is encountered. However, in practical applications there are always either true multicomponent analyte mixtures or analytes with unwanted traces of spurious residues. Here we focus on modeling the response of a plasmonic sensor surrounded by a binary mixture of adsorbates.

The adsorption process in practical applications is truly multicomponent, truly nonlinear and truly stochastic. The mandatory method of the stochastic analysis, based on the master equation for the probabilities that the system has a given composition as a function of time, i.e., chemical master equation (CME) [28], gives the complete insight into the stochastic behavior of the system over time. However, it is seldom the case that the CME related to nonlinear systems can be solved analytically. There are numerical solutions, based on CME, proposed by Gillespie et al, developed and improved over time, also implemented into a standalone software solution for multiple platforms (StochKit2) [29-37]. These algorithms for simulation of stochastic reaction kinetics, called stochastic simulation algorithms (SSA), differ from the analytical approach for developing and forming CME. Beside including stochastic instantaneous numbers of adsorbate molecules on the surface, they also take into account time as a stochastic variable. The residential time of an adsorbed particle on the surface has an exponential distribution and numerical calculations rely on the use of built-in random number generators in a specific programing environment. We illustrate the advantages and the pitfalls of the SSA in the text.

In order to allow for multiscale modeling of more complex systems we focus here on the features of various approaches to modeling binary adsorption. We first give some theoretical background of plasmonic sensing of adsorbed analytes, then we consider the criteria for the validity of equations based on the linear first order kinetics of single- 
component adsorption. After that we deal with numerical solvers with the aim to generalize the criteria to binary adsorption and address the stochastic behavior by applying the stochastic simulation algorithms written for binary adsorption. Finally we deal with training an ANN capable of providing us with the steady state values of the adsorbed amount of particular analytes of a binary mixture in plasmonic sensors. We give the obtained functions along with the corresponding subroutines as an open source material suitable for further use by other researchers. We also expect our results to be scalable and therefore suitable to be generalized to mixtures of an arbitrary number of adsorbates.

\section{Theoretical approach based on analytical solutions}

In general, surface phenomena of adsorption and desorption take place at the interface between two different phases: liquid/gas, solid/gas, solid/liquid [2,38]. We refer here to adsorption of fluid particles on solid surfaces capable of supporting surface plasmon polaritons - SPP (metals or other plasmonic materials) [39]. This situation corresponds to various kinds of refractometric affinity sensors, from the conventional SPP sensors based on evanescent wave propagation at the interface between a semi-infinite dielectric and a semi-infinite metal to complex nanostructured plasmonic metamaterial-based sensors.

The benefits of optical readout with surface plasmon polaritons (SPP) detection are multiple. The operation of plasmonic sensors does not require the tagging of analyte particles as in fluorescence detection [40], they can operate in real time, their sensitivity is extremely high [41], their all-optical detection is way faster than electronic, thus ensuring monitoring of nano-second dynamics [42]. Plasmonic sensors are refractometric, i.e. they are based on the reading of changes in refractive index caused by the presence of analytes. The SPP waves were first observed in 1902 as an "anomaly" (the existence of unexpected black lines) in the spectrum of light reflected from a diffraction grating located on a metal surface [43], later explained [44,45]. This has been since exploited in many applications. The SPP waves are surface bound, their intensity decreases exponentially with distance from it (evanescent waves). All electromagnetic changes at the surface, that is, at the point of establishment of SPP waves, directly affect the readable optical parameters at the interface, which is therefore ideal for detecting and monitoring surface processes. The surface plasmon polariton moves in the plane between the medium with a negative value of relative dielectric permittivity, $\varepsilon<0$ (metal or other material with free electron plasma) and the medium with a positive value, $\varepsilon>0$ (dielectric, or analyte-containing analyte) at a frequency close to plasmonic resonance. When the analyte is adsorbed on the interface, it modulates the SPP wave propagation conditions and thus changes the refractive index $n$. This change is read from the outside by an interrogating light beam.

Figure 1. shows a schematic diagram of the operation of plasmonic sensors in three different scales. Optical detection of the reflected beam from the surface, presented as red lines on the left side of the picture, follows the evanescent field of an excited SPP beam on the sensor surface (shown as a pink line and its spatial distribution being shown in the inset at the lower left of the picture). The surface may be solid plasmonic material, functionalized by depositing a thin layer of a ligand in order to enable selective binding of a targeted receptor. Alternatively, it may be nanoengineered (thus forming a metasurface) in order to tailor the reflectance spectrum as shown in the middle. In a red ellipse on the right, the picture shows a surface with adsorbed molecules surrounded by free moving ones. 
The equations that relate kinetic constants - the rate constant for the adsorption and the rate constant for the desorption - with the parameters on a molecular level such as the desorption energy, molecular projection area and mass, depend on the application. The development of the equations in [27] is based on the ideal gas theory and provides a complete analytical solution of the rate constants. In biosensing applications the surrounding medium is liquid and the determination of rate constants relies on experimental or numerical methods [46,47]. The software solution for calculations of rate constants based on the known desorption energy and molecular constants is available at Mendeley Data, the repository with open access [48] and the datasets with desorption energies and molecular constants based on experimental data acquired from literature is given on Harvard and Mendeley Data repositories [49-51].

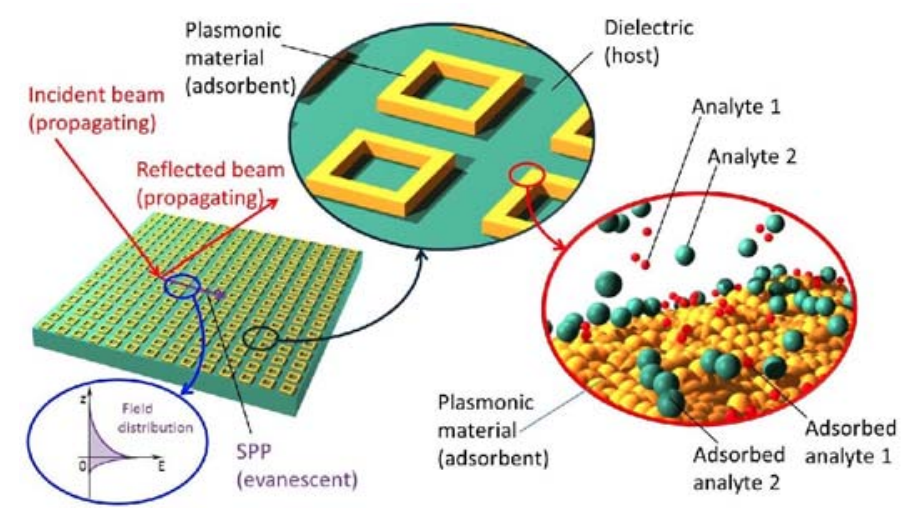

Fig. 1 Schematic presentation of competitive adsorption of binary mixture on a metamaterial surface

The stoichiometric equation that describes the binary adsorption shown in the Fig. 1 is

$$
A_{g, i}+A_{f} \underset{k_{d i}}{\stackrel{k_{a i}}{\rightleftarrows}} A_{a, i}, \quad i=1,2
$$

where $A_{g}$ refers to free fluid molecules, i.e. molecules in the gas or liquid phase, $A_{f}$ refers to the free adsorption centers on the plasmonic surface and $A_{a}$ refers to the molecules adsorbed on the surface. $k_{a}$ is the adsorption rate constant and $k_{d}$ is the desorption rate constant. $i$ is an index denoting the adsorbate species 1 or 2 .

In general case, which assumes taking into account the depletion of the analyzed sample due to analyte adsorption (closed reaction chamber where analyte is not repleted), the reaction rate equation (RRE) that governs the kinetics of this system is

$$
d N_{i} / d t=k_{a i}\left(N_{0 i}-N_{i}\right)\left(M-N_{1}-N_{2}\right)-k_{d i} N_{i}, i=1,2
$$

$N_{0}$ is the total number of analyte particles (both those available in the sample for adsorption and those already adsorbed), $M$ is the number of adsorption centers on the plasmonic surface, $N$ is the instantaneous number of particles adsorbed on the surface, and $V_{a d s}$ and $V_{d e s}$ are adsorption and desorption rates, respectively. Equation set (2) is a set of nonlinear (second order) differential equations of Riccati type. It can be written as a matrix and form the matrix Riccati differential equation (MRDE). The solutions to MRDE in a closed form have not been found so far [52-54]. There are results on solving MRDE by the use of neural networks [55], applicable for nonlinear singular systems. 
When the sample depletion is neglected, the RRE has the form of a linear (first order) differential equation, Lagergren equation

$$
d N_{i} / d t=k_{a i} N_{0 i}\left(M-N_{1}-N_{2}\right)-k_{d i} N_{i}=V_{a d s L}-V_{d e s L}, i=1,2
$$

The related refractive index change, $R I C$, caused by the changes in the adsorbed amount, is estimated as a weighted sum of refractive indexes of the adsorbed particles, where the weight coefficients $w_{i}$ are calculated based on the difference between the refractive indexes of condensed analytes, $n_{a}$, and the refractive index of the environment, $n_{e}$, which is in accord with the effective medium theory - simple mixing rule [56]:

$$
R I C=\sum_{i=1}^{2} N_{i}\left(n_{a i}-n_{e}\right) / M=\sum_{i=1}^{2} w_{i} N_{i}
$$

First we address here the criteria for the validity of approximate modeling of the refractive index change due to monocomponent adsorption and then we consider the possibilities for generalization and the establishment of the criteria for the validity of approximate modeling of the refractive index change due to adsorption of binary mixtures.

\subsection{Affinity sensor's response due to monocomponent adsorption}

The first criterion for the validity of approximate modeling of the refractive index change due to monocomponent adsorption by the linear model

$$
N_{0}>M
$$

stems directly from the comparison of the Lagergren RRE (linear model) and the Riccati RRE (nonlinear model)

$$
\begin{gathered}
d N / d t=k_{a}\left(N_{0}-N\right)(M-N)-k_{d} N=V_{a d s}-V_{d e s} \\
d N / d t=k_{a} N_{0}(M-N)-k_{d} N=V_{a d s L}-V_{d e s L}
\end{gathered}
$$

respectively. $V_{a d s(L)}$ and $V_{\text {des }(L)}$ are the adsorption and desorption rates. If the overall number of molecules/particles $N_{0}$ is sufficiently large, its decrease due to adsorption can be neglected and the reaction rate equation reverts from a Riccati differential equation to a Lagergren-type equation.

However, second-order reactions can be studied by the PFO (pseudo first order) kinetics not only when the condition (5) is satisfied, but also when another criterion is met. The second condition revealed in [11] is

$$
k_{d} / k_{a} \gg 2\left(N_{0}+M\right)
$$

The conditions (5) and (7) can be expressed in the form suitable for the adsorption based gas sensing applications

$$
p_{1} \leq p \leq p_{2}, p_{1}=k_{B} M T / V, p_{2}=v_{0} e^{-E_{d} /(R T)} \sigma_{S} \sqrt{\pi m_{0} k_{B} T / 2} / \alpha-k_{B} M T / V
$$

where $k_{B}$ is the Boltzmann constant, $m_{0}$ is the mass of a single adsorbate molecule, $V$ is the reaction chamber volume, $T$ is temperature, $E_{d}$ is the desorption energy of adsorbate molecules, $R$ is the universal gas constant, $\alpha$ is the sticking coefficient, $\sigma$ is the surface density of adsorption centers, and $v_{0}$ is the vibration frequency of the crystal lattice.

The solutions to reaction rate equations (RRE) for monocomponent adsorption process are known for both models (Riccati and Lagergren). The time evolution of the number of adsorbed molecules is an exponential regression towards a steady state. When the conditions (5) and (7) are met, the time evolutions of the number of adsorbed particles coincide, and when they are not satisfied, the time evolutions differ in a steady state and/or the time constant, i.e. in the transient time needed to reach the steady state. 
In a steady state (equilibrium) the adsorption rate equals the desorption rate and in spite of the ongoing adsorption and desorption on the plasmonic surface the number of the adsorbed molecules and the percentual surface coverage remain constant. Figure 2 represents a graphical interpretation of the first condition (5) for the approximate equality of the steady-state numbers of adsorbed particles predicted by the two models, $N_{e L}$ and $N_{e}$, the greater the difference $N_{0}-M$, the closer the values of the stationary states calculated in accordance to the linear and the nonlinear RRE.

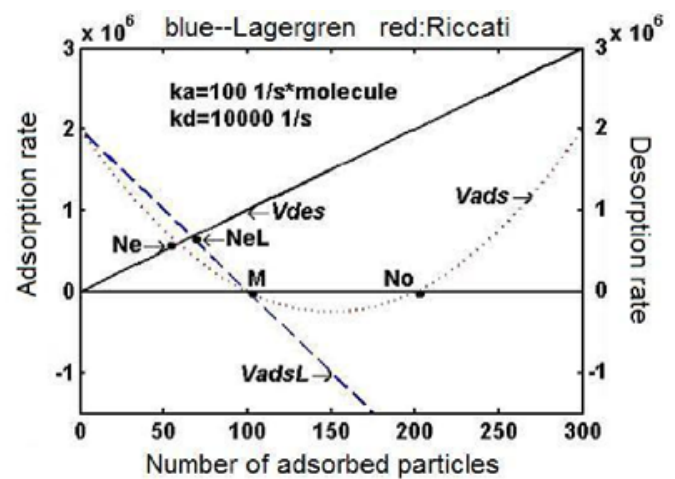

Fig. 2 Graphical representation of the first condition. When Eq. (5) is valid, the stationary states $N_{e}$ and $N_{e L}$ calculated according to Riccati and Lagergren RRE respectively, are close to each other. $V_{a d s L}$ is the adsorption rate in Lagergren RRE, $V_{a d s}$ is the adsorption rate in Riccati RRE, $V_{d e s}$ is the desorption rate, the same in both RRE, $M$ is the number of adsorption centers on the surface, $N_{0}$ is the overall number of adsorbate molecules.

Figures 3 and 4 show graphically the reasoning behind applying the second condition (7). Namely, if the desorption rate is steep (Fig. 4), desorption dominates, and since it is modeled in the same way in both models, the instantaneous differences in adsorption rates can be neglected.

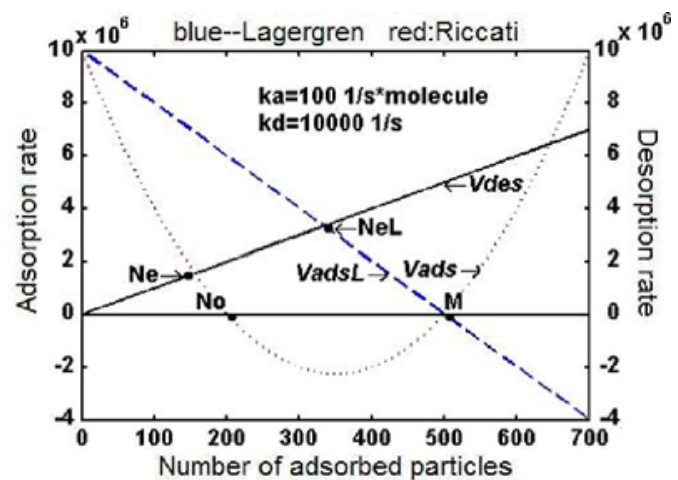

Fig. 3 The first condition, Eq. (5) is not valid - stationary states for the linear and nonlinear model differ. Notation as in Fig.2. 
Figures 2, 3 and 4 show clearly that if linear modeling is employed inappropriately, the number of the adsorbed number of molecules in equilibrium is overestimated, $N_{e L}$ is always greater than $N_{e}$.

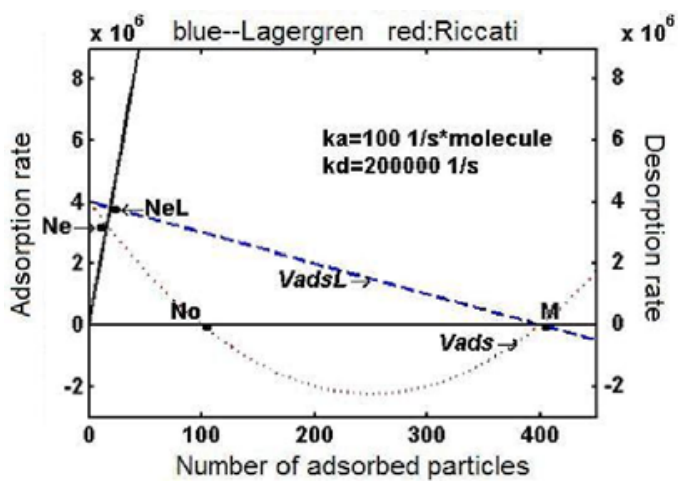

Fig. 4 The first condition [Eq. (5)] is not valid but stationary states are close. Notation as in Fig.2

A graphical interpretation of the conditions (8), aimed to support the proper modeling of gas adsorption at the plasmonic structures, is shown in Fig. 5. The condition linearly dependent on temperature but independent on the gas species is represented by a solid black line. At pressures above that line the model based on Lagergren kinetics is valid and below the line it is not. The second condition additionally depends on the gas species (i.e. on $E_{d}$ ). This is represented by the curves above which the linear model is valid, while below them it is not. According to [11], if any of the conditions is met, the results based on linear and nonlinear RRE are virtually the same - it is sufficient that at least one of the conditions is fulfilled for the linear approximations to be valid. The intuitively clear conclusion, that the more accurate model becomes necessary at low pressures and low temperatures, can be quantified in practical applications by using Fig. 5. It is illustrative that in applications related to gas sensing, modeling based on the linear RRE is likely to be the first choice.

The kinetics of adsorption in refractometric adsorption-based chemical sensing is usually written in terms of concentration. The two conditions for determining the domain of applicability for monocomponent adsorption can be interpreted in terms of the reciprocal value of the equilibrium constant, i.e. $k_{d} / k_{a}$ ratio, and the binding capacity, Figure 6. The binding capacity, the analyte concentration and the reciprocal equilibrium constant are given in the convenient $M$ units (moles per liter). Whenever the binding capacity is comparable with the analyte concentration or greater than it and the reciprocal equilibrium constant (the ratio $k_{d} / k_{a}$ ) is smaller than the double sum of the binding capacity and analyte concentration (the area below the convex surface in Fig. 6), the model based on Lagergren kinetics is not appropriate.

According to literature data [57] the rate constants of adsorption in optical biosensors used for recognition of biological macromolecules have a very broad range. For instance Canziani [57] reported the concentrations of analyte in range from 2 to $30 \mathrm{nM}$, the binding capacity of the ligand $333 \mathrm{M}$ and the dynamic range for the reciprocal equilibrium constants calculated according to the reported rate constants for the particular analyte- 
ligand pairs (human interleukin IL-5, SIL-5Ralpha) was from 2.3 to $4.8 \mathrm{nM}$. Hence, in many such reactions the nonlinear model is likely to be the first choice.

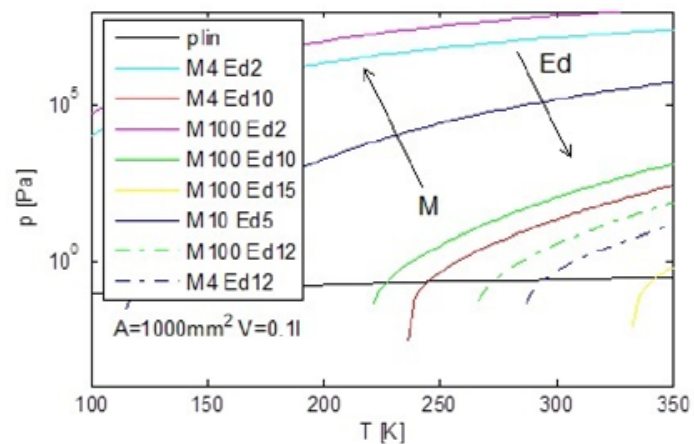

Fig. 5 Applicability limits for Lagergren modeling of plasmonic gas detection for various combinations of molar masses $M$ and desorption energies $E_{d}$. The number of the adsorption centers on the surface is $6.25 \cdot 10^{14}$, the molar mass is given in $\mathrm{g} / \mathrm{m}^{3}$, the desorption energy is in $\mathrm{kcal} / \mathrm{mol}$ and the reaction chamber volume is $1 \mathrm{dm}^{3}$. The linear model is not applicable under the black solid line denoted as "p lin" and above the colored solid and dash-dotted curves.

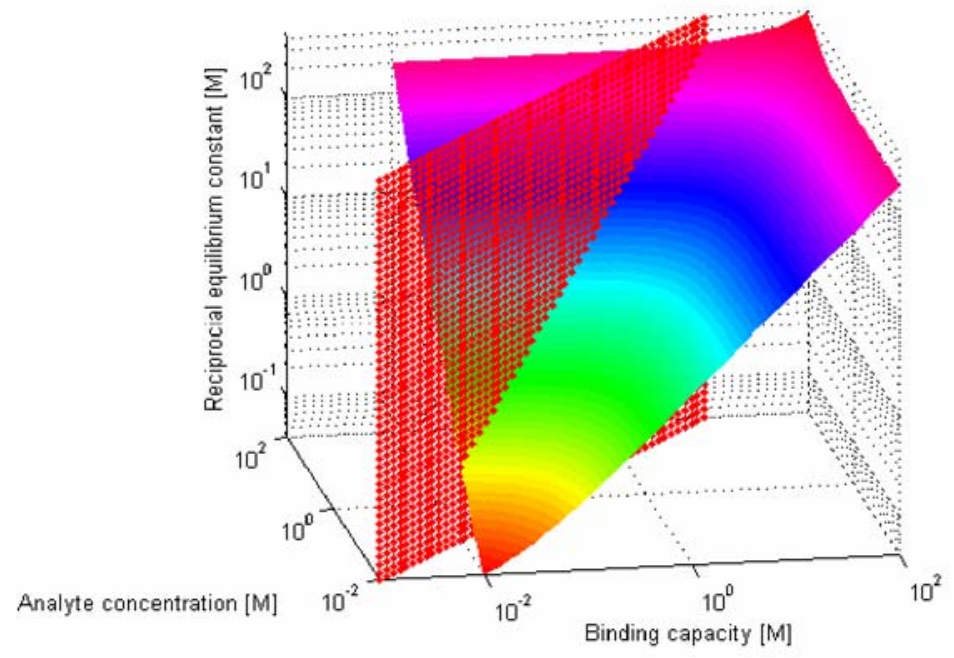

Fig. 6 Applicability limits for the calculations of the response of adsorption based plasmonic sensor for analytes with known rate constants.

The solutions to Eq. (6a) and Eq. (6b) are

$$
N(t)=\frac{M k_{a} N_{0}}{k_{d}+k_{a} N_{0}}\left(1-e^{-\left(k_{d}+k_{a} N_{0}\right) t}\right)
$$




$$
\begin{aligned}
& N_{a}(t)=\beta \frac{1-e^{-k t}}{1-(\beta / \gamma) e^{-k t}}=\beta+\sum_{i=1}^{\infty}(\beta-\gamma)\left(\frac{\beta}{\gamma}\right)^{i} e^{-i k t} \\
& \beta=\left[\left(N_{0}+M+k_{d} / k_{a}\right)-\sqrt{\left(N_{0}+M+k_{d} / k_{a}\right)^{2}-4 N_{0} M}\right] / 2 \\
& \gamma=M N_{0} / \beta
\end{aligned}
$$

Visually, they have the same form, the exponential regression towards the steadystate. In [57] another criterion for the validity of the pseudo first order kinetics is proposed. It is based on observation of the response, i.e. on the fitting of experimental results. Treated that way, the domain of validity of the linear model is even broader than bounded by limits presented so far. But, before generalizing towards modeling the binary adsorption, we remark here that in fitting the nonlinear response with one transient, one time constant, one gets mathematical form resembling the form of the linear response but the obtained numerical values will differ from the values in the linear model itself.

\subsection{Affinity sensor response to binary adsorption}

The solution to the linear RRE set is known for the affinity sensor response to multicomponent adsorption in general, for an arbitrary mixture [25]

In the case of binary adsorption with the first order kinetics, (3) is a set of two coupled equations, the response function of each component in the mixture consisting of a constant describing the stationary response and of two vanishing transients. These transients are weighted differently but have the same time constants:

$$
\begin{aligned}
& N_{1}(t)=\frac{M k_{a 1} N_{01} k_{d 2}}{z_{1} z_{2}}+\frac{M k_{a 1} N_{01}\left(k_{d 2}+z_{1}\right)}{z_{1}\left(z_{1}-z_{2}\right)} e^{z_{1} t}-\frac{M k_{a 1} N_{01}\left(k_{d 2}+z_{2}\right)}{z_{2}\left(z_{1}-z_{2}\right)} e^{z_{2} t} \\
& N_{2}(t)=\frac{M k_{a 2} N_{02} k_{d 1}}{z_{1} z_{2}}+\frac{M k_{a 2} N_{02}\left(k_{d 1}+z_{1}\right)}{z_{1}\left(z_{1}-z_{2}\right)} e^{z_{1} t}-\frac{M k_{a 2} N_{02}\left(k_{d 1}+z_{2}\right)}{z_{2}\left(z_{1}-z_{2}\right)} e^{z_{2} t}
\end{aligned}
$$

The notation is as before $-z_{1}$ and $z_{2}$ are the roots of a polynomial obtained by transforming two coupled first order ordinary differential equations (ODE) with two variables into one second order ODE with one variable.

$$
\begin{aligned}
& z_{1,2}=\left(-b \pm \sqrt{b^{2}-4 c}\right) / 2 \\
& b=k_{a 1} N_{01}+k_{d 1}+k_{a 2} N_{02}+k_{d 2} \\
& c=k_{a 1} N_{01} k_{d 2}+k_{a 2} N_{02} k_{d 1}+k_{d 1} k_{d 2}
\end{aligned}
$$

In general, due to linearity and the fact that the numbers of adsorbate molecules obey the multinomial distribution, the deterministic solution and all higher moments may be represented in terms od the first moment (expectation), the number of transients in the response of any component of the mixture equals the number of components in the mixture and all components have transients with the same time constants.

The nonlinear RRE set that governs the binary adsorption with the second order kinetics, modeled by Eq. (2), is not solved in an analytical form. However, the solution for stationary states is known [27]:

$$
N_{1 s}^{3}+u_{2} N_{1 s}^{2}+u_{1} N_{1 s}+u_{0}=0, \quad N_{2 s}=\frac{k_{d 1} k_{a 2} N_{02} N_{1 s}}{k_{d 2} k_{a 1} N_{01}+\left(k_{d 1} k_{a 2}-k_{d 2} k_{a 1}\right) N_{1 s}}
$$




$$
\begin{gathered}
u_{0}=\frac{k_{a 1} k_{d 2} M N_{01}^{2}}{k_{d 1} k_{a 2}-k_{a 1} k_{d 2}}, \quad u_{2}=\frac{k_{d 2} k_{a 1} N_{01}+k_{d 1} k_{a 2} N_{02}}{\left(k_{d 1} k_{a 2}-k_{d 2} k_{a 1}\right)}-M-N_{01}-\frac{k_{d 1}}{k_{a 1}} \\
u_{1}=M N_{01}-N_{01} \frac{k_{d 2} k_{a 1}\left(N_{01}+M\right)+k_{d 1} k_{a 2} N_{02}+k_{d 1} k_{d 2}}{\left(k_{d 1} k_{a 2}-k_{d 2} k_{a 1}\right)}
\end{gathered}
$$

The index $s$ refers to the stationary value. It is also known that in general, the stationary state of one component in the mixture that follows the nonlinear equation set during adsorption represents a root of the polynomial of the degree greater by one than the number of the components in the mixture [27]. Hence in the binary case it is the cubic polynomial. For every found root of a polynomial, all other stationary states are calculated in terms of that found one, so mathematically, there are multiple stationary states. In [58] it is proved that only one set of stationary states in binary adsorption has physical meaning, is real and realistic but in implementing this result a correct selection of the appropriate solution is still needed, which may require non-negligible processing time.

\section{Computer aided solutions}

We address here numerical solvers, fitting of the experimental results, stochastic simulation algorithms and the response analysis assisted by trained artificial neural networks.

\subsection{Numerical solvers and fitting of the experimental results}

The results obtained by numerical solvers are application-specific, valid for the set of parameters used in calculations. However, they lack the generality that characterizes analytical solutions. Nevertheless, they are more and more prominent in multiscale modelling. The computing performances are constantly improving and the pool of available software constantly increases.

There are pitfalls of automated calculations using the same set of coding routines over a very wide range of parameters. One is the propagation of numerical errors. One way to deal with it, independent of the used software platform, is to adapt analytical expressions to make them less vulnerable to numerical errors. For instance, the expression for the stationary state in (9b) is vulnerable but can be computed from the other root by the use of Vieta's formulas [59]. Another problem is that the parameters used may change the nature of the equations. For instance, the set of differential equations (2) may become stiff or unstable. Additional information is then needed to facilitate the propper selection of physically meaningful results. In this research, that information was drawn from the conclusions on stability of binary adsorption in [58]. Any oscillatory response is excluded from the pool of numerical results.

In general, direct solvers (for example MathWorks COMSOL's PARDISO, MUMPS) provide solutions generated by using fixed steps (hence encountering memory problems when scaling down the steps) while iterative solvers provide one with adaptive steps but setting them up manually requires a lot of expertise. In order to avoid calculation stability issues and problems with convergence it is advisable to split the independent calculations and segregate complex routines if possible.

For the calculations used to obtain the results presented here, Mathworks MATLAB R13 software environment was used, and the routines from the package [48], for solving the system of nonlinear differential equations Dormand-Prince method of order 4 and 
Bogacki-Shampine method of order 3 were applied. Concurrent calculations were performed in Octave and MathWorks COMSOL environments. Fitting was performed by using the solution in [60]. The codes used, along with the obtained results are available from [61].

An exemplary result regarding the validity of the approximate linear modeling of binary adsorption is shown in Fig. 7.

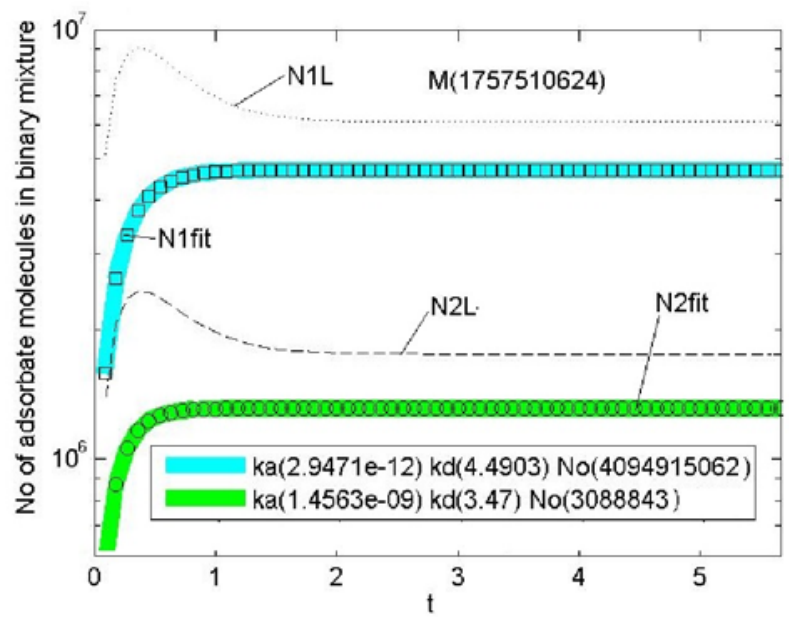

Fig. 7 Number of adsorbate molecules of binary mixture over time calculated in three ways: by the use of a numerical solver (blue wide line and green wide line), by fitting the numerical solution (squares and circles) and by the use of an analytical expression valid for the first order kinetic reactions (dotted and dashed lines), for the components 1 and 2 , respectively.

This result is in agreement with the criteria for validity of the pseudo first order kinetic model of monocomponent adsorption from [10]. We applied here the generalized version of those criteria and fitted curves searching for pair of solutions that has, for each component, one stationary value and two transient values. However, contrary to the real linear model where transients have the same set of time constants, here transients have two independent time constants each. The starting points for search for them are first the time constants of the linear model. Then, when searching for the time constants in the response of the other component in the mixture the starting points are the time constants found for the first component. The satisfactory result may be surprisingly simple. In this particular example, the calculated time constants of the linear model are $0.287812 \mathrm{~s}$ and $0.2221 \mathrm{~s}$; the calculated time constants for the component 1 in the Riccati set of equations are 0.22171 and 0.22171 , i.e. they are identical; and calculated time constants for the component 2 in the Riccati set of equations are 0.16760 and 0.167456 , very close to each other and different from the time constant of the first component.

The fact that there is an example where the true response of a separate component in the mixture behaves as if all transients but one can be neglected, although due to nonlinearities supposedly it has an infinite number of transients is very important. It opens possibilities for new speculations on the kinetics of the process (such as an explanation of the mixture behavior where one part reaches the equilibrium faster than the other). It also paves the way for further investigations towards the appropriate simplifications of adsorption modeling that is a part of more complex systems. 


\subsection{Stochastic simulation of binary adsorption in plasmonic sensors}

The adsorption process is stochastic in nature. The instances of the approach of molecules to the surface, their residential time on the surface and the departure time from the surface are all random. Hence the instantaneous number of adsorbed molecules on the surface is also random. A true stochastic approach to numerical modeling of chemical kinetics, proposed by Gillespie [30,36] is used in many applications. This algorithm is computationally demanding, and the real challenge is an efficient implementation of the SSA. Many adaptations on the original SSA have been developed so far, one of them is the accelerated exact stochastic simulation (AESS) [62].

Figure 8 is obtained by the standard, unaccelerated procedure, code and generated data are available from [61].

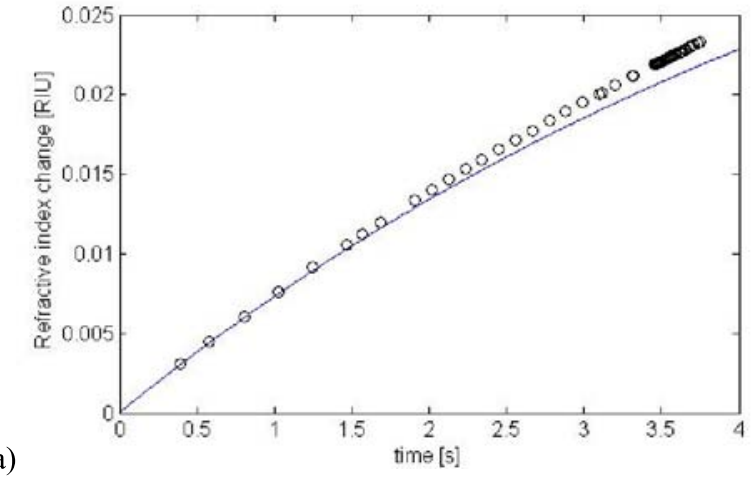

a)

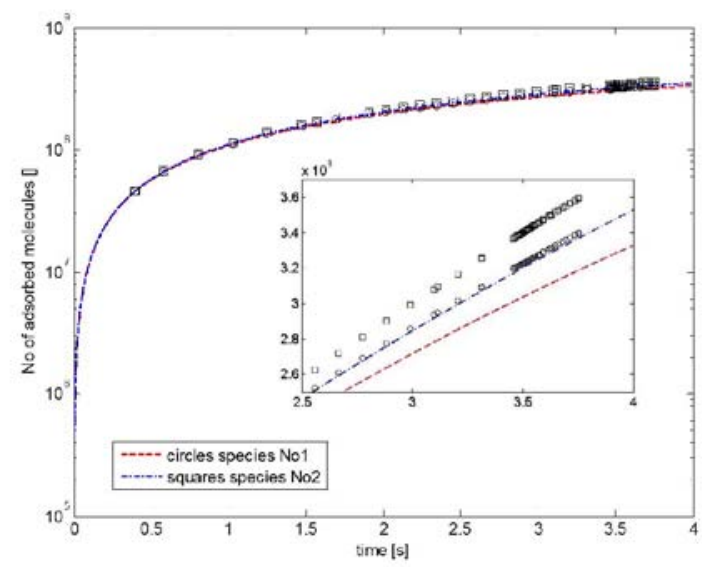

b)

Fig. 8 a)The refractive index change and b) the number of adsorbed molecules of two components in binary mixture over time calculated by the use of the stochastic simulation algorithm (symbols) and by numerically solving RRE (lines) 
Parameters used for calculations are as follows: the vector of adsorption rate constants is $\left[1.33 \cdot 10^{-11} ; 1.33 \cdot 10^{-12}\right]$; the vector of desorption rate constants is $\mathrm{kd}=[0.08 ; 0.08]$; the vector of the number of molecules of components present in the system is $\mathrm{No}=\left[3 \cdot 10^{9}\right.$; $3 \cdot 10^{10}$ ]; and the number of adsorption sites on the surface is $\mathrm{M}=3 \cdot 10^{-9}$.

The example is chosen to illustrate how computationally expensive this algorithm is. The data shown in picture (circles and squares) are selectivelly taken from the original set of generated data which occupies $10 \mathrm{~Gb}$ space in over $500 \mathrm{csv}$ files each having over 1500 lines [61]. The steps for reproducing the stochastic response are consecutive, the propensity functions for each transition are calculated using the instantaneous numbers of adsorbed molecules on the surface. All reactions are performed for an interval of length $\tau$ before updating the propensity functions needed for the calcuation of the next time step. One way to overcome this disadvantage is $\tau$-leaping method, the approximate method implemented by updating the adsorption rates less often. This sometimes allows for more efficient simulation and thus the consideration of larger systems but can diverge from the solution obtained by numerical solvers, as shown in the inset in Fig. 8b. Results can be obtained faster by trained artificial neural networks (ANN).

\subsection{ANN-based tool for the estimation of the steady-state response}

It is noted that ANNs perform better than other methods, especially in highly nonlinear cases. Also, this technique can build an efficient model using a small set of data, but its accuracy improves with larger data sets. Generating models by a neural network requires a large number of iterative calculations and depending on computer resources the computation time can be long.

Here we focus on the determination of the equilibrium values for binary adsorption based on machine learning algorithms. The result is MATLAB function, obtained by training a neural network on numerical experiments. For input formed of parameters of the reaction rate equations, the function calculates the equilibrium values of the adsorbed amount of the components in the case of a binary mixture. For training of the artificial neural network we used the Neural Net Fitting application provided within the framework of MathWorks MATLAB software.

Initial steps: generating database for training the network

Our database emerged as a result of over 1000 numerical experiments performed in Octave, release 4.4.1 and 500 numerical experiments performed in MATLAB, release R2015a.

The results of numerical experiments are stored in Microsoft Excel as csv files and used as inputs for training the ANN. After generating a database of numerical experiments, we apply Neural Net Fitting and analyze fitting results.

Training the $A N N$

The Neural Net Fitting application provided within the framework of MathWorks MATLAB is based on multi-layer perception (MLP).

The Neural Network used three layers - the input layer, the hidden layer and the output layer, where the input layer consists of different number of inputs variables/parameters. The Back Propagation Algorithm (BPA) is used as stochastic approximation to nonlinear regression. The structure of the neural networks structures designed and used here is $7-\mathrm{xx}-2$, with 7 corresponding to the number of input layer 
neurons, $\mathrm{xx}$ to the number of hidden layer neurons and 2 to the number of output layer neurons. The change in number of hidden layer neurons directly affects the results. Among several networks whose number of hidden layer neurons belongs to the set of values $(7-25)$ one is chosen as optimal. The block diagram of the chosen ANN with the best performance is given in Fig. 9. The MLP architecture consists of an input layer, 20 hidden layers and one output layer. The hidden layer uses a hyperbolic tangent sigmoid transfer function.

The input layer consists of 7 input nodes, parameters of the RRE, ka1 ka2 kd1 kd2 No1 No2 and M matrix or vector data. The output layer has two nodes and they correspond to the steady-state values of the particular analytes in a binary mixture. The applied training algorithm is based on the Bayesian regularization. The data for network is divided into three categories: training, validation and testing. It was set so that $70 \%$ of the total data are used for the ANN training process, $15 \%$ of the data are used for validation and $15 \%$ of data for the testing process.

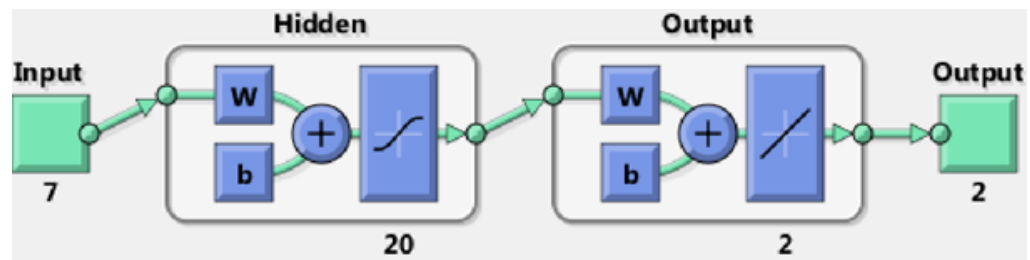

Fig. 9 The details of the block diagram of the ANN used in this study. $\mathrm{x} 1=[\mathrm{ka} 1 \mathrm{ka} 2 \mathrm{kd} 1 \mathrm{kd} 2 \mathrm{No} 1 \mathrm{No} 2 \mathrm{M}]$, output should deliver the steady-state values of the particular analytes in a binary mixture

The target is calculated in accord with the exact solution for the response of the steady-state in the process of binary adsorption. The output corresponds to the values obtained by the trained ANN. The neural network developed by the use of 275 sets of data had a high accuracy for the output prediction. $\mathrm{R}$ values were 0.97914 and 0.819695 for training and testing respectively and the corresponding MSE values were $4.16992 \cdot 10^{-3}$ and $5.18747 \cdot 10^{-2}$.

After aditional training MSE value droped to $1.12822 \cdot 10^{-2}$ and $R$ became 0.946554 . Figure 10 shows the results that correspond to training, testing and overall results. Figure 11 shows a comparison between the output calculation using a linear fit and the ANN values of output for particular components in the binary mixture. The results of the ANN prediction output show good agreement with the values from numerical experiments. 

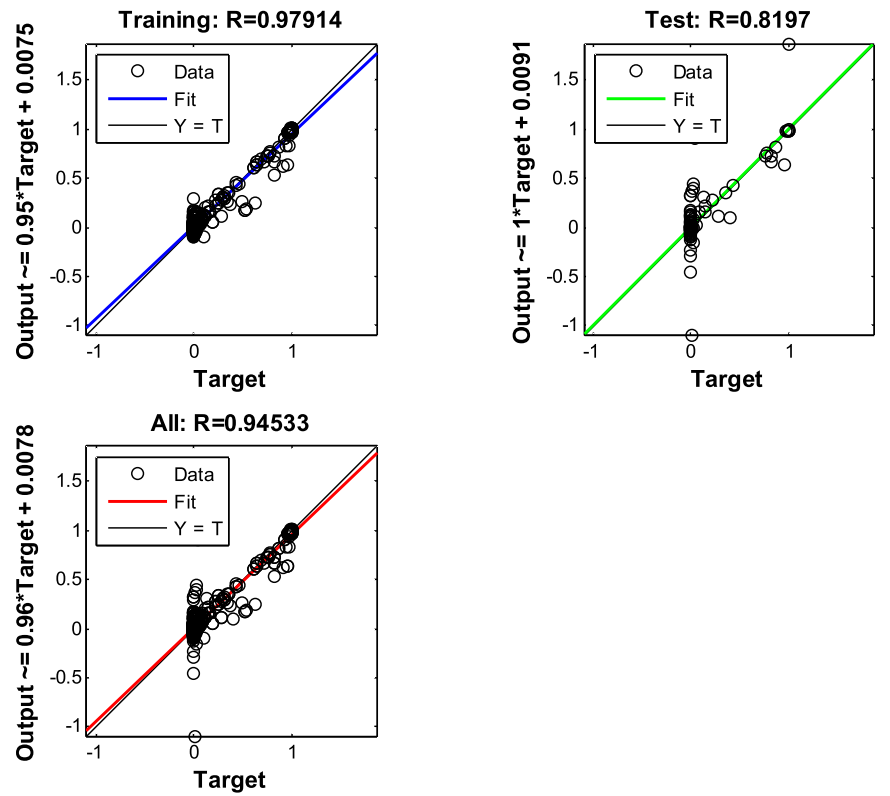

Fig. 10 The results that correspond to a) training, b) testing and c) overall results.

a)

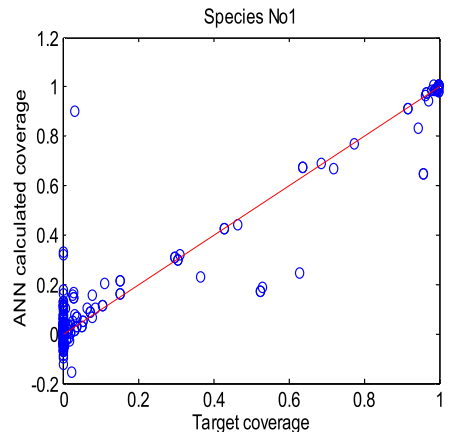

b)

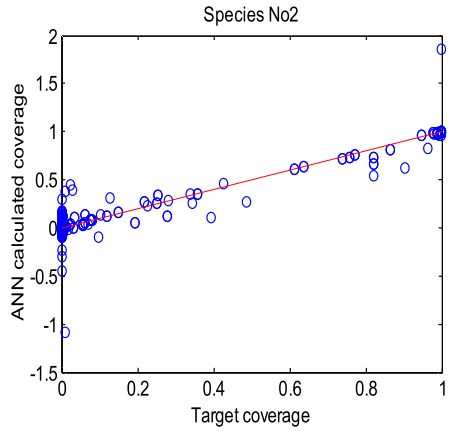

Fig. 11 Comparison of exact values and values estimated by the use of artificial neural network for a) the first component in the binary mixture and for the b) the other component in the binary mixture

Figure 12 shows testing of the obtained function on a new set of data. The results are moderate, compared to good results from [24], where training was performed not by using the Bayesian regularization algorithm but by using the quasi-Newton BFGS (BroydenFletcher-Goldfarb-Shanno) algorithm instead. Further exploitation of the created function, its adaptation for the use of other algorithms and creating new functions with the same ANN and new data sets are enabled by the use of codes given in open source Mendeley Database [63]. 

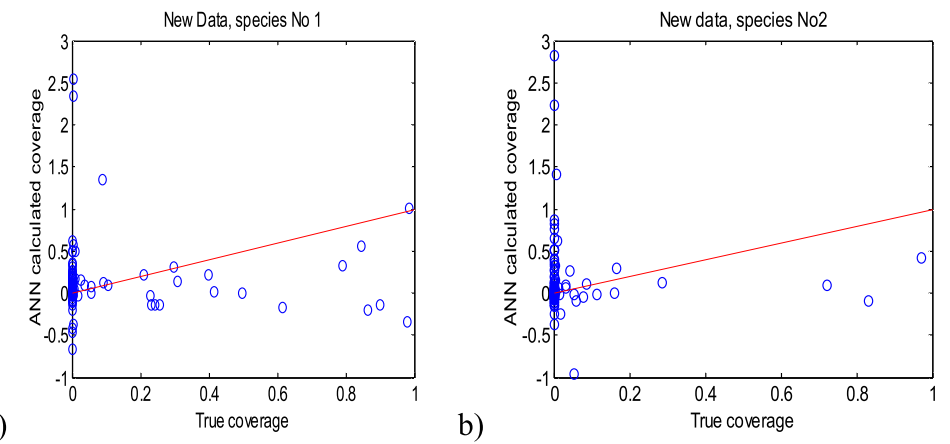

Fig. 12 Comparison of the exact values and the values estimated by the use of artificial neural network for a) the first component in the binary mixture and for the b) the other component in the binary mixture

\section{Discussion and conclusion}

The paper addresses binary adsorption-desorption process modeled with coupled first order differential equations or with coupled second order differential equations that can be reverted to the first order differential equations if certain conditions are met. The paper gives a review of the criteria developed for modeling the refractive index change in plasmonic sensors by the monocomponent linear adsorption model.

The criteria for the choice between these two models for the case of single-component adsorption are given in graphical form and also used for generalization, addressing the criteria for the validity of usage of these two models for the case of binary adsorption.

The paper presents the advantages and pitfalls of the use of various numerical techniques for numerical modeling of the kinetics of binary adsorption process such as using numerical solvers, fitting the experimental results, the use of stochastic simulation algorithms and machine learning in artificial neural networks (ANN). The ANN that calculates adsorption amounts in equilibrium trained by using the Levenberg-Marquardt and the Bayesian regularization algorithms proved to be less efficient than the one trained by the use of the quasi-Newton BFGS (Broyden-Fletcher-Goldfarb-Shanno) algorithm.

Besides their application in plasmonic chemical sensing, the presented results can be useful for modeling of the adsorption induced noise in general adsorption-based devices and for modeling the kinetics of two-component ligand-receptor binding, optical reading of interactions of biological molecules, for modeling the time response of adsorption based devices with optical readout in various applications such as gas detection, monitoring of interactions of biomolecules, etc.

All data and codes needed for the intermediate and final results in this paper are available on digital repositoria with open access (Mendeley Data and Harvard Dataverse).

Acknowledgments This work is a part of the research funded by the Serbian Ministry of Education, Science and Technological Development. 


\section{References}

1. W. J. Thomas and B. Crittenden, Adsorption Technology and Design (Elsevier Science \& Technology Books, 1998).

2. J. Toth, Adsorption: Theory, Modeling, and Analysis, surfactant (Marcel Dekker Incorporated, 2002).

3. A. Dabrowski, Adv. Colloid Interface Sci. 93, 135 (2001).

4. L. Zhou, Adsorption: Progress in Fundamental and Application Research (World Scientific, 2007).

5. A. B. Dahlin, B. Dielacher, P. Rajendran, K. Sugihara, T. Sannomiya, M. ZenobiWong, and J. Vörös, Anal. Bioanal. Chem. 402, 1773 (2012).

6. S. Y. Lagergren, Zur Theorie Der Sogenannten Adsorption Gelöster Stoffe (Kungliga Svenska Vetenskapsakademiens, Hadlingar, 1898).

7. R. L. Tseng, F. C. Wu, and R. S. Juang, J Taiwan Inst Chem E 41, 661 (2010).

8. Y. Liu and L. Shen, Langmuir 24, 11625 (2008).

9. H. Qiu, L. Lv, B. Pan, Q. Zhang, W. Zhang, and Q. Zhang, J. Zhejiang Univ. Sci. A 10, 716 (2009).

10. W. Stroberg and S. Schnell, Math. Biosci. 287, 3 (2017).

11. O. Jakšić, I. Jokić, Z. Jakšić, Ž. Čupić, and L. Kolar-Anić, Phys. Scr. 2014, 014047.1 (2014).

12. F. Amato, J. L. González-Hernández, and J. Havel, Talanta 93, 72 (2012).

13. Y. W. Huang, M. Q. Chen, and Q. H. Li, J. Therm. Anal. Calorim. 138(1), 451 (2019).

14. E. Weinan, Principles of Multiscale Modeling (Cambridge University Press, 2011).

15. Z. W. Ulissi, M. T. Tang, J. Xiao, X. Liu, D. A. Torelli, M. Karamad, K. Cummins, C. Hahn, N. S. Lewis, T. F. Jaramillo, K. Chan, and J. K. Nørskov, ACS Catal. 7, 6600 (2017).

16. J. L. González-Hernández, M. M. Canedo, and S. Encinar, J. Math. Chem. 51, 1634 (2013).

17. J. L. González-Hernández, M. M. Canedo, and S. Encinar, MATCH Commun. Math. Comput. Chem. 79, 619 (2018).

18. G. V. S. R. Pavan Kumar, K. A. Malla, B. Yerra, and K. Srinivasa Rao, Appl. Water Sci. 9, 44 (2019).

19. M. R. Fagundes-Klen, P. Ferri, T. D. Martins, C. R. G. Tavares, and E. A. Silva, Biochem. Eng. J. 34, 136 (2007).

20. R. Anderson, J. Rodgers, E. Argueta, A. Biong, and D. A. Gómez-Gualdrón, Chem. Mater. 30, 6325 (2018).

21. B. Gezer, U. Kose, D. Zubov, O. Deperlioglu, and P. Vasant, Wirel. Netw. June (2019) https://doi.org/10.1007/s11276-019-02035-1.

22. M. Ghaedi, M. Roosta, A. M. Ghaedi, A. Ostovan, I. Tyagi, S. Agarwal, and V. K. Gupta, Res. Chem. Intermed. 44, 2929 (2018).

23. S. Ullah, M. A. Assiri, A. G. Al-Sehemi, M. A. Bustam, M. Sagir, F. A. Abdulkareem, M. R. Raza, M. Ayoub, and A. Irfan, Int. J. Environ. Res. 14, 43 (2020).

24. H. Barki, L. Khaouane, and S. Hanini, Kem. u Ind. 68, 289 (2019).

25. O. M. Jakšić, Z. S. Jakšić, Ž. D. Čupić, D. V. Randjelović, and L. Z. Kolar-Anić, Sensors Actuators B Chem. 190, 419 (2014).

26. O. Jakšić, Ž. Čupić, Z. Jakšić, D. Randjelović, and L. Kolar-Anić, Russ. J. Phys. Chem. A 87, 2134 (2013).

27. O. M. Jakšić, D. V Randjelović, Z. S. Jakšić, Ž. D. Čupić, and L. Z. Kolar-Anić, Chem. Eng. Res. Des. 92, 91 (2014). 
28. C. W. Gardiner, Handbook of Stochastic Methods for Physics, Chemistry and the Natural Sciences (Springer Berlin-Heidelberg-New York, 1985).

29. D. T. Gillespie, A. Hellander, and L. R. Petzold, J. Chem. Phys. 138, 170901 (2013).

30. D. T. Gillespie, J. Phys. Chem. 81, 2340 (1977).

31. D. T. Gillespie, J. Comput. Phys. 22, 403 (1976).

32. D. T. Gillespie, J. Chem. Phys. 115, 1716 (2001).

33. S. Lampoudi, D. T. Gillespie, and L. R. Petzold, J. Chem. Phys. 130, 094104 (2009).

34. D. T. Gillespie, J. Chem. Phys. 113, 297 (2000).

35. D. T. Gillespie, J. Phys. Chem. A 106, 5063 (2002).

36. D. T. Gillespie, Annu. Rev. Phys. Chem. 58, 35 (2007).

37. H. Li, Y. Cao, L. R. Petzold, and D. T. Gillespie, Biotechnol. Prog. 24, 56 (2008).

38. D. D. Do, Adsorption Analysis: Equilibria and Kinetics (Imperial College Press, London, 1998).

39. S. Maier, Plasmonics. Fundamentals and Applications (Springer Science + Business Media, 2007).

40. A. Felinger, J. Chromatogr. A 1184, 20 (2008).

41. L. S. Jung, C. T. Campbell, T. M. Chinowsky, M. N. Mar, and S. S. Yee, 7463, 5636 (1998).

42. C. L. Byard, X. Han, and S. B. Mendes, Anal. Chem. 84, 9762 (2012).

43. R. Wood, Philos. Mag. 4, 396 (1902).

44. H. Raether, Springer Tracts Mod. Phys. 111, 1 (1988).

45. E. Kretschmann and H. Raether, Z. Naturforsch. 23, 2135 (1968).

46. M. S. Mehand, G. De Crescenzo, and B. Srinivasan, J. Mol. Recognit. 25, 208 (2012).

47. M. S. Mehand, B. Srinivasan, and G. De Crescenzo, Sci. Rep. 5, (2015).

48. O. Jakšić, Mendeley Data http://dx.doi.org/10.17632/R9YT6TJZNJ.1 (2018).

49. O. Jakšić, Mendeley Data http://dx.doi.org/10.17632/w78xsxmg8d.1 (2019).

50. O. Jakšić, Mendeley Data http://dx.doi.org/10.17632/pwsc2jgwzk.1 (2019).

51. O. Jakšić, Harvard Dataverse (2018).

52. G. Freiling, G. Jank, and H. Abou-Kandil, Linear Algebra Appl. 241-243, 291 (1996).

53. M. A. Lohe, J. Math. Phys. 60, (2019).

54. G. Freiling, Linear Algebra Appl. 241-243, 291 (1996).

55. J. A. Samath and N. Selvaraju, IJCA Int. J. of Comput. Appl. 1, 48 (2010).

56. T. C. Choy, Effective Medium Theory: Principles and Applications (Oxford University Press, 2016).

57. G. Canziani, W. Zhang, D. Cines, A. Rux, S. Willis, G. Cohen, R. Eisenberg, and I. Chaiken, Methods A Companion to Methods Enzymol. 19, 253 (1999).

58. O. M. Jakšić, Z. Jakšić, M. B. Rašljić, and L. Z. Kolar-Anić, Adv. in Math. Phys. 2019, 1 (2019).

59. T. Stanković, N. Dalarsson, and O. Jakšić, in 4th Symp. Math. Appl. 24. i 25. Maj 2013, 99 (2014).

60. M. Holmes, fits $(f, x d, y d, p 0, n, m)$ (MATLAB Cent. File Exch. Retr. March 31 2020) https://www.mathworks.com/matlabcentral/fileexchange/72113-fits-f-xd-yd-p0-n-m 61. O. Jakšić, Mendeley Data http://dx.doi.org/10.17632/gfv2vzpx3p.1 (2020).

62. D. Jenkins and G. Peterson, Mendeley Data http://dx.doi.org/10.17632/n3s9f96jwp.1 (2011).

63. O. Jakšić, Mendeley Data http://dx.doi.org/10.17632/2jdnx2pfys.1 (2020). 